\title{
Diagnóstico diferencial de los transtornos temporomandibulares (T. T. M).
}

Resins used in the manufacture of provisional prothesis. Case report.
As resinas utilizadas na fabricação de temporários. Reporte de um caso.
Fecha de Recepción

27 de junio de 2013
Aceptado para su publicación

25 de octubre de 2013
Carmen Collante

Profesora Adjunta a cargo

Cátedra de Ortodoncia. FOUNNE.

E-mail: carmencollante@hotmail.com

\section{Omar Álvarez}

Profesor Titular. Cátedra Oclusión. FOUNNE.

E-mail: oalvarez@odn.unne.edu.ar

Ricardo Hugo Altamirano

Auxiliar docente de $I^{a}$ categoría. Dedicación exclusiva. Cátedra Oclusión. FOUNNE.

E-mail: rhaltamirano@odn.unne.edu.ar

Claudio Modenutti

Auxiliar docente de $I^{a}$ categoría. Dedicación exclusiva. Cátedra Semiología. FOUNNE.

E-mail: camodenutti@odn.unne.edu.ar

\section{Mariana Osnaghi}

Becaria de investigación.

Auxiliar docente de $I^{a}$ categoría.

Cátedra Clínica de Prótesis I Curso. FOUNNE.

E-mail: mosnaghi@odn.unne.edu.ar

\section{Resumen}

Cualquier alteración en los componentes de la Articulación Temporo Mandibular (ATM), genera un desorden, el cual presenta signos evidentes y puede ser o no asíntomatico.

A los efectos de establecer un diagnostico presuntivo de los Trastornos Témporo Mandibulares, el "Mapa del dolor" creado por el Dr Mariano Rocabado, permite a través de la división topográfica de las A.T.M en 8 sectores la posibilidad de identificar diferentes trastornos $y$ anomalías en su funcionamiento.

Este procedimiento clínico permite identificar e interceptar un problema articular en sus inicios, siendo un elemento de diagnóstico precoz, como así también un método preventivo de Trastorno Temporo Mandibular. Orienta al clínico en el enfoque terapéutico hacia su resolución paliativa o definitiva y permite ver la evolución del tratamiento.

Para confirmar un diagnostico presuntivo sobre las posibles disfunciones lo ideal es la realización de una Resonancia Magnética Nuclear en la posición adecuada y otros estudios complementarios.

\section{Palabras claves}

Mapa deldolor,Sinovial,Hiperlaxitud, Retrodisco.

\section{Abstract}

Any change in the components of the Temporomandibular Joint (TMJ), generates a 
disorder, which showing obvious signs and may be asymptomatic or not.

For the purpose of establishing a presumptive diagnosis of TMD, the "pain map" created by Dr. Mariano Rocabado, allowed through topographical division of the TMJ in 8 sectors the ability to identify different disorders and abnormal operation.

This clinical procedure allows identifying a joint problem in the beginning, being an element of early diagnosis, as well as a preventive method of TMD. It guides the clinician in the therapeutic approach to palliative or definitive resolution and can see the evolution of treatment.

To acknowledge a presumptive diagnosis of possible malfunctions, ideally conducting is to do a Nuclear Magnetic Resonance in the proper position and other studies.

\section{Keywords}

Map of pain, Synovial, Hypermobility, Retrodisco.

\section{Sumário}

Qualquer alteração nos componentes da Articulação Temporomandibular (ATM) gera uma desordem, as quais apresentam sinais evidentes e pode ser assintomático ou não.

Com a finalidade de estabelecer um diagnóstico presuntivo de DTM, o "mapa da dor", criado por Dr. Mariano Rocabado, permitiu através da divisão topográfica da ATM em 8 setores a capacidade de identificar diferentes transtornos e operação anormal.

Este procedimento clínico permite identificar um problema comum no início, sendo um elemento de um diagnóstico precoce, bem como um método de prevenção de DTM. Ele orienta o clínico na abordagem terapêutica para a resolução paliativo ou definitivo e pode ver a evolução do tratamento.

Para confirmar o diagnóstico presuntivo de possíveis avarias idealmente a realização de uma ressonância magnética na posição adequada e outros estudos.

\section{Palavras chave}

Mapa da dor,Sinovial,Hipermobilidade, Retrodisco.

\section{Introducción}

A los efectos de establecer, un diagnostico presuntivo de los Trastornos Temporo Mandibulares,el "Mapa del dolor" creado por el Dr Rocabado Mariano, permite a través de la división topográfica de las A.T.M en 8 sectores la posibilidad de identificar diferentes trastornos, anomalías en su funcionamiento, $y$ orientar el tratamiento hacia su resolución paliativa o definitiva.

Dicho procedimiento requiere un operador entrenado en la percepción semiológica de estos desordenes y sus posibles causas.Las causaspueden ser intracapsulares y extracapsulares .Entre las intracapsulares,se encuentran los desplazamientos discales, los trastornos retrodiscales, - desordenes de los músculos masticadores (mialgias),el mapa del dolor ayuda a identificar estas causas.

El objetivo de este trabajo es proveer al práctico general una herramienta útil para la evaluación funcional de la A.T.M, basada en un protocolo semiológico simple en donde la palpación y la aplicación de cargas orientan a descubrir los vectores de cargas que provocan patologías al sistema estomatognático.

\section{Desarrollo}

\section{A. Características anatómicas de la Articulación Temporo Mandibular (ATM)}

La ATM es una articulación formada por el cóndilo del hueso de la mandíbula con la cavidad glenoidea y el cóndilo del hueso temporal. Entre ellos existe un cartílago sin osificar: Disco Articular .Este genera dos compartimentos entre el cóndilo mandibular y el cóndilo del temporal en forma de saco denominados cavidades sinoviales. El sistema neurovascular lo constituyen el nervio maseterino y auriculo temporal y ramas de la Carótida externa y maxilar interna.

Toda la ATM está rodeada de una cápsula articular fibrosa, la cara lateral de esta cápsula es más gruesa y se llama ligamento temporomandibular.

Los cóndilos temporal y mandibular son los únicos elementos activos participantes en la dinámica articular por lo que se considera a esta articulación una diartrosis bicondílea'. 
REVISTA FACULTAD DE ODONTOLOGÍA ISSN No 1668-7280 - Vol. VI № 2 - 2013

47
DIVULGACIÓN

Diagnostico diferencial de los transtornos temporomandibulares (T. T. M).

\section{B. Trastornos más comunes de la ATM y sus causas}

El desplazamiento del Disco es una patología frecuente que padece hasta un $20 \%$ de la población,en la mayoría de los casos no requiere tratamiento y la única sintomatología es el ruido articular ${ }^{3}$. El $80 \%$ de la población mundial padece de algún tipo de disfunción en las A.T.M, las mujeres en un $75 \%$ son las más afectadas, de cada 5 personas, 3 son mujeres en la que prevalece éstas patologías.

Osteoartritis de la ATM, anquilosis, fracturas del cuello o cabeza del cóndilo.

Las causas más comunes pueden ser4: microtrauma a repetición (más frecuente), parafunción, bruxar, malos hábitos,oclusión dental.

Los Macrotraumas: golpes, fracturas, posición de boca abierta prolongada o sostenida en el tiempo (extracción de terceros molares, endodoncias).

Traumatismos de alta velocidad: movimiento rápido, violento o involuntario de la mandíbula, originado por una fuerza externa (whiplash).

\section{Mapa del Dolor}

El mapa del dolor articular es un procedimiento clínico desarrollado por el Dr. Rocabado que permite evaluar en forma rápida y efectiva la sensibilidad dolorosa de los tejidos blandos articulares mediante la identificación de los componentes blandos (ligamentos) y espacios (por ej zona bilaminar).

Facilita el diagnostico de diversas patologías que la afectan identificando diferentes trastornos o anomalías en su funcionamiento a partir de saber sus componentes y sus posibles dolores, de acuerdo al lugar donde se manifiestan.

Esta técnica, nos permite estudiar la ATM a través de su división topográfica en 8 sectores.

Entre las principales ventajas del mapa del dolor articular podemos resaltar:

- Permite identificar un problema articular en sus inicios. Es un excelente elemento de diagnóstico precoz, como también un método preventivo de desórdenes temporomandibulares.

- Orienta o guía al clínico hacia un enfoque terapéutico.

- Permite detectar el dolor en puntos articulares específicos y bien definidos anatómicamente.

- Permite realizar en todas las sesiones la evaluación y ver la evolución del tratamiento.

\section{Técnica semiológica}

La articulación se divide en 8 zonas las cuales identifican al elemento anatómico, y a través de la palpación y manipulación semiológica, se establece un diagnostico presuntivo sobre las posibles disfunciones. (Ver tabla I)

Esta técnica consiste en hacer compresión o distensión delicada de las 8 zonas anatómicas de la articulación intra (Sinovial) y extracapsular (ligamentosa) para luego evaluar al paciente.

Los 8 puntos básicos pueden apreciarse en la figura $n^{\circ}$ I ellos son:

\section{Sinovial Antero Inferior}

2. Sinovial antero Superior

3. Ligamento colateral lateral

4. Ligamento temporomandibular

5. Sinovial póstero inferior

6. Sinovial póstero superior

7. Ligamento posterior

8. Retrodisco

Cada ATM es palpada en forma independiente, a través de una secuencia de pasos que no demanda mucho tiempo,si se motiva adecuademente al paciente, una vez dominada la técnica. En las primeras tres zonas el examen se realiza por fuera de la cavidad bucal,como se observa en la figura $n^{\circ} 2$,en las zonas restantes el examen es intra y extra bucal,como se puede apreciar en la figura $n^{\circ} 3$.

\section{Posición inicial de examen secuencial} La posición inicial de examen debe ser en protrusión y con boca abierta porque en protrusiva el cóndilo baja y se desplaza hacia lateral lo que hace más fácil su palpación, y a 10 milímetros debido a que a esa apertura las sinoviales están descomprimidas, es como una posición de reposo para estas. Se debe tratar de mantener estable la posición inicial de examen mientras se realiza la técnica del Mapa del Dolor.

\section{Instrucciones al paciente}

Instruirlo a que cada vez que perciba aumento 
REVISTA FACULTAD DE ODONTOLOGÍA ISSN No 1668-7280 - Vol. VI № 2 - 2013

48
DIVULGACION

Collante - Álvarez - Altamirano - Modenutti - Osnaghi

\begin{tabular}{|c|l|}
\hline ZONA & POSIBLES DISFUNCIONES \\
\hline 1 & Hipermovilidad articular (hiperlaxos) \\
\hline 2 & Apretador o I osteofito que produce compresión \\
\hline 3 & Desplazamiento antero medial del disco (ligamento colateral externo elongado) \\
\hline 4 & Hiper laxitud del ligamento o retracción capsular. \\
\hline 5 & $\begin{array}{l}\text { Compresión de la zona posterior (sinovial) clase } 2 \text { con fulcrum del mismo lado y produce } \\
\text { desplazamiento cóndilodiscal. }\end{array}$ \\
\hline 6 & $\begin{array}{l}\text { Pérdida de la D. V desdentados, posteriores - braqui severos, desarmonias oclusales, } \\
\text { apretadores. }\end{array}$ \\
\hline 7 & Apretamiento, clases II, ${ }^{a}$ División. \\
\hline 8 & Aumento presión intrarticular - apretador (bruxista) disminuye D. V posterior. \\
\hline
\end{tabular}

de su molestia o aparición de dolor, levante una mano como señal visual para el clínico. Si es solo una molestia lo que se percibe bastará con levantar levemente la mano, pero si es dolor el paciente deberá elevarla más, siendo esta acción proporcional a la magnitud de este. El procedimiento debe llevarse a cabo con fineza y suavidad.

\section{Procedimiento ${ }^{7,8}$}

\section{Zona I: Sinovial Antero Inferior}

Localizada I I/2 cm por delante y I cm por debajo del trago. Deslizar el dedo índice desde el polo lateral hasta ubicar el polo anterior e inferior del cóndilo, palpar el tejido hasta sentir una dureza. La prueba positiva $(+)$, es decir molestia o dolor indica:

- Hipermovilidad condilar anterior con patrón mandibular protrusivo o contralateralrepetitivo. - Proceso de apertura bucal exagerada, donde el cóndilo comprime la superficie de la membrana sinovial. El dolor sinovial ánteroinferior o dolor I corresponde a la condición inicial de un proceso disfuncional hacia hipermovilidad condilar. Por esto mismo, representa el signo más nosotros detectamos y que paradojalmente pasa desapercibido y no se describe como una alteración propiamente

\section{Zona 2: Sinovial Antero Superior}

Se localiza desde la ubicación anterior desplazando el dedo índice hacia arriba y adelante $1 \mathrm{~cm}$, (debajo de la apófisis cigomática). Si la prueba es $(+)$, indica traslación condilar más allá del borde inferior de la eminencia articular. Esta condición es un signo claro de hipermovilidad anterior.

\section{Zona 3: Ligamento Colateral Lateral}

En apertura máxima $(45 \mathrm{~mm})$, se la encuentra desplazando el dedo índice de abajo hacia arriba ejerciendo presión moderada, sobre el polo condilar externo. Si el test el (+) indica sobredistensión ligamentosa, la que se puede deber a hipermovilidadcondilar y/o a una posición del disco hacia medial (luxación o subluxación).

Terminado el punto 3 se pide al paciente que degluta, cambie de posición la cabeza y repose 15 segundos.

\section{Zona 4: Ligamento Temporo Mandibular}

Para evaluar este punto el clínico debe pedir al paciente una apertura bucal media,de $20 \mathrm{~mm}$, apoyar el pulgar sobre las caras oclusales de las piezas dentarias póstero inferiores del lado a examinar y ejercer presión hacia atrás y abajo poniendo en tensión. Realizar esta maniobra hasta sentir la tensión final ligamentosa, la sensación es de tope.

Si la prueba es $(+)$, se debe interpretar que el cóndilo a adoptado una posición pósteroinferior con la consecuente sobre elongación ligamentosa y perdida de la congruencia de las superficies articulares.

Una condición crónica no diagnosticada puede 
crear una distensión irreversible del ligamento perpetuando una patología articular de inestabilidad. Es necesario recordar que el ligamento es un elemento no elástico.

Terminado el punto 4 se pide al paciente que degluta, cambie de posición la cabeza y repose 15 segundos.

\section{Zona 5: Sinovial Postero Inferior}

Corresponde a la zona posterior del cóndilo,zona del retro disco, Desde la posición inicial de examen y una vez ubicado el polo lateral del cóndilo deslizar el dedo a la región posterior y descender hasta el cuello del cóndilo. Si hay dolor implica que el cóndilo se encuentra en posición distal provocando un efecto de microtrauma sobre la sinovial pósteroinferior.

\section{Zona 6: Sinovial Postero Superior}

Se la reconoce, con la boca semiabierta 20 $\mathrm{mm}$.Palpar el borde posterior del cóndilo y deslizar el dedo hacia craneal hasta sentir el techo de la fosa temporal y el borde posterior del cóndilo. Presionar en forma suave y gradual hasta sentir el contacto con tejido duro. Si hay dolor implica que el cóndilo está comenzando a adoptar una posición posterior y superior sobre la porción posterior densa del disco. El cóndilo en forma indirecta, a través del polo posterior del disco, comprime la sinovial posterosuperior contra la fosa.

\section{Zona 7: Ligamento Posterior}

Corresponde a zona bilaminar de la ATM, la mandíbula en la misma forma descrita en la zona 4 y a continuación, realizar movimientos suaves y cortos en sentido de adelante hacia atrás y hacia arriba con el propósito de relajar la cápsula articular y que esta no oponga resistencia a la movilización condilar. Logrado esto, desplazar la mandíbula en sentido posterior hasta sentir el tope óseo. Luego con la mano libre aplicada sobre la región del ángulo goníaco, ejercer una fuerza en sentido craneal. El objetivo de esto es provocar una sobrecarga sobre el borde posterior denso del disco. Si la prueba es (+), es probable que el cóndilo se esté apoyando sobre una zona articular no apta para soportar presión. Se debe interpretar que el cóndilo a adoptado una posición posterosuperior con un desplazamiento

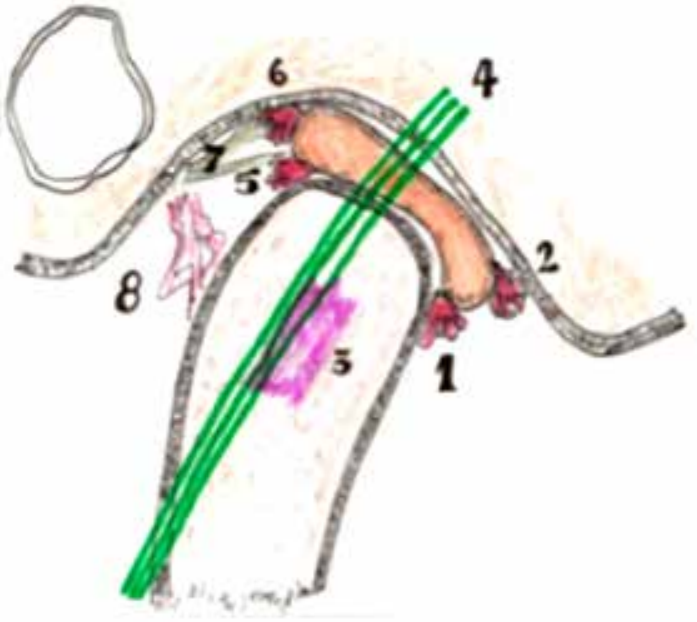

Figura I. Gráfico de Zonas del Mapa del dolor.

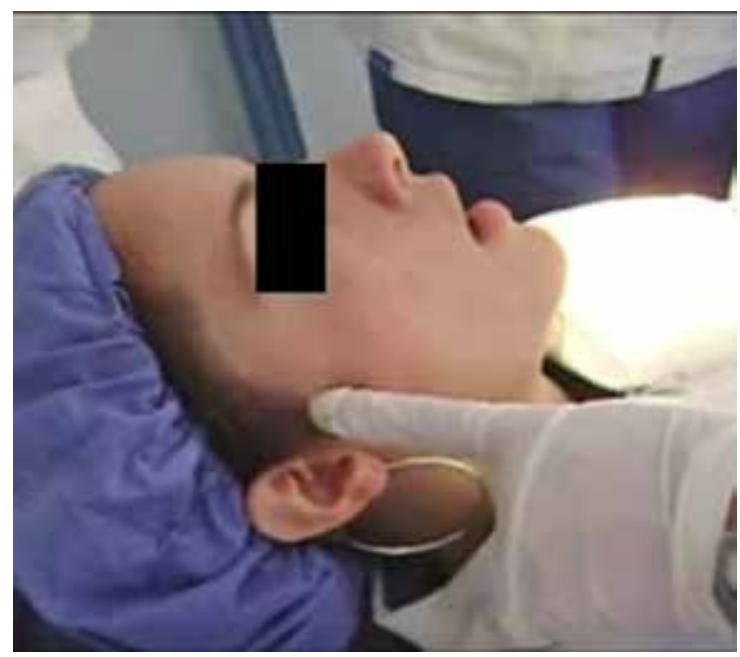

Figura 2. Palpación Zona 2 (sinovial superior, región anterior) ${ }^{7}$.

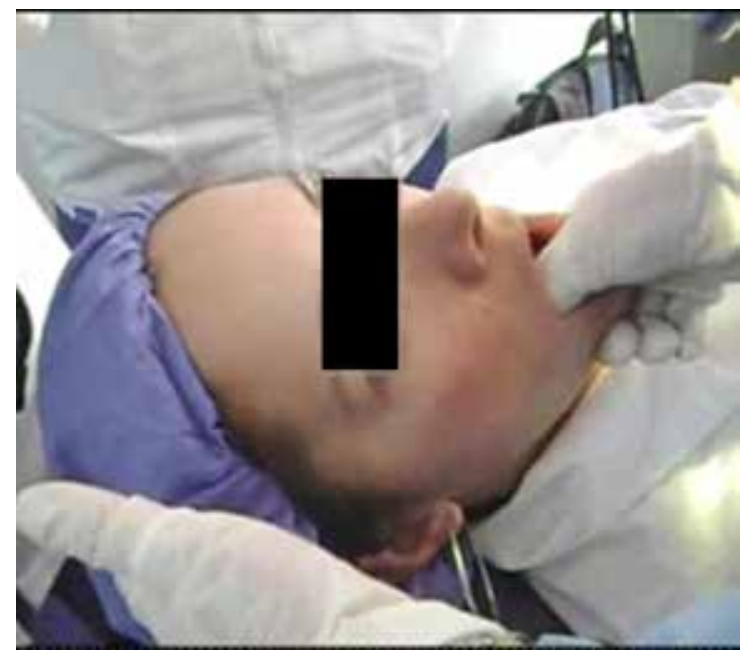

Figura 3. Maniobra para identificar vectores de carga craneal-caudal'. 
REVISTA FACULTAD DE ODONTOLOGÍA

ISSN No 1668-7280 - Vol. VI № 2 - 2013

50
DIVULGACIÓN

Collante - Álvarez - Altamirano - Modenutti - Osnaghi anterior del disco y pérdida de la congruencia de las superficies articulares,por lo que la lesión se considera intracapsular.

\section{Zona 8: Retrodisco}

Retrodisco corresponde a la inserción del ligamento posterior a la zona posterior y densa del disco, es decir, es la unión entre ambas estructuras. Para su evaluación colocar las manos igual que para la zona anterior y llevar la mandíbula adelante con precaución ya que si la prueba es $(+)$ estamos frente a un proceso de retrodiscitis. Esta situación se caracteriza por inflamación con sangrado, muy doloroso e invalidante y con limitación funcional a la masticación y apertura.

Para confirmar un diagnostico presuntivo sobre las posibles disfunciones de las 8 zonas de la ATM lo ideal es la realización de una Resonancia Magnética Nuclear en la posición adecuada y otros estudios. Por ejemplo para saber si hay sinovitis (Zonas I, 2, 5 y 6) la RMN se debe pedir en T.2 solamente.

\section{Conclusiones}

Este protocolo de inspección de la ATM, otorga al clínico una orientación de la probable disfunción, pero es imprescindible contar con el apoyo de las imágenes de la resonancia magnética para determinar el diagnostico preciso que con lleve a instalar el tratamiento adecuado. Por otra parte, brinda la posibilidad de comunicar al paciente, si la patología puede ser resuelta, o simplemente impedir su progreso y agravamiento. No todos los desórdenes tienen resolución definitiva, a su vez, hay que tener un enfoque multidisciplinario combinando el uso de fármacos, tratamientos kinésicos y odontológicos.

\section{Bibliografía}

I. Campos A. Rehabilitación oral y oclusal. Vol I. España: Editorial Harcourt; 2000.

2. Alonso A. Albertini J. y Bechelli A. Anatomía aplicada de la articulación temporomandibular. Oclusión y diagnóstico en rehabilitación oral. I $^{\mathrm{a}}$ ed $2^{\mathrm{a}}$ reimpresión. Argentina: Editorial Panamericana; 2003.

3. Okesson J. P. Diagnóstico de los trastornos temporomandibulares. Tratamiento de Oclusión y afecciones temporomandibulares. Ed 6ta. Barcelona (España): Editorial Elsevier; 2008.

4. Ash M. Ramfjord S. Oclusión. $4^{\mathrm{a}}$ ed. México: Editorial McGraw Hill Interamericana; 1996.

5. Posselt U. y Mallat E. Fisiología de la oclusión y rehabilitación. $2^{\mathrm{a}}$ ed. Barcelona (España): Edit Jims; 1973

6. Rocabado M. Cabeza y cuello: tratamiento articular. Buenos Aires: Intermédica editorial. 1979.

7. Video: Mapa del Dolor de la ATM. Disponible en http://www.youtube.com/watch?v=lxh8ulJzrps. Consultado en Junio 2013.

8. Mapa Del Dolor Articular. Disponible en http://www.buenastareas.com/ensayos/Mapa-Del-Dolor-Articular/48347/3. htm. Consultado en Junio 2013. 